\title{
1. Non-Linear Theory of an Equatorial Flow. I
}

\author{
By Koji Hidaka, M. J. A. \\ Ocean Research Institute, Tokyo University, Tokyo
}

(Communicated Oct. 12, 1979)

Introduction. In the present paper will be discussed a method to compute a steady, oceanic flow in which the velocity components (VC) and pressure gradients have no variation in east-west direction. In the equatorial zones of the oceans, particularly in the Pacific and Indian Oceans, this condition is approximately fulfilled.

In the previous paper, ${ }^{4)}$ a method of dynamical computation of ocean currents was given in a meridional section of the Pacific, considering the Coriolis forces, pressure gradients, and the friction due to both vertical and lateral mixing. In the computation the authors could locate an eastward flow comparable in magnitude with equatorial undercurrent. A few years later, Arthur ${ }^{1)}$ pointed out that $v(\partial u / \partial y)$ will amount to $10^{-4}$ on both sides of the equator and will no longer be negligible.

In another paper, ${ }^{3)}$ it was discussed to compute the velocity of flow at the equator, considering the inertia terms $-v(\partial u / \partial y)$ and $-v(\partial v / \partial y)$ respectively, and the result was compared with that obtained from the method proposed by Hidaka ${ }^{2)}$ and Tsuchiya ${ }^{5)}$ almost simultaneously for computing the velocity at the equator itself. By this comparison, we could have an idea on the influence of the inertia terms on the magnitudes of an equatorial flow.

The problem. Suppose a meridional section passing through the equator. Take $x$-, $y$ - and $z$-axis positive east-, north- and down-ward, and let $\mathrm{VC}$ in these directions be $u, v$ and $w$ respectively. Assume that the east-west variation of $\mathrm{VC}$ is small compared with the meridional, and neglect $u(\partial u / \partial x)$ and $u(\partial v / \partial x)$.

Since it is not easy to solve non-linear equations as they stand and it is well-known that the ocean current is in most cases represented by geostrophic flows except at or close to the equator, we shall neglect the frictional forces due to mixing in dynamical equations. Then the steady state equations for a planetary flow will become

$$
2 \omega \sin \varphi v-v \frac{\partial u}{\partial y}-\omega \frac{\partial u}{\partial z}=\frac{\partial D}{\partial x} ;-2 \omega \sin \varphi u-v \frac{\partial v}{\partial z}-\omega \frac{\partial v}{\partial z}=\frac{\partial D}{\partial y},
$$

where $D$ is a geopotential distance of an isobaric surface counted above a sufficiently deep reference level, $w$ the angular velocity of the Earth and $\varphi$ the geographical latitude. 
The general principle of solving (1) is first to neglect:

$$
-w(\partial u / \partial z) \text { and }-w(\partial v / \partial z)
$$

and to solve the ordinary non-linear simultaneous equations:

$$
2 \omega \sin \varphi v-v \frac{\partial u}{\partial y}=\frac{\partial D}{\partial x} ; \quad-2 \omega \sin \varphi u-v \frac{\partial v}{\partial y}=\frac{\partial D}{\partial y} .
$$

The solution will be possible if (2) are small compared with $\partial D / \partial x$ and $\partial D / \partial y$ respectively. The principal part of this paper is devoted for solving (3).

When the approximate values of $\mathrm{VC}$ are computed along the meridional section, it will be possible to compute approximately the vertical velocity $w$ from the equation of continuity. This enables us to compute (2), by subtracting these from $\partial D / \partial x$ and $\partial D / \partial y$, we obtain (3), there being slight changes in the magnitudes of $\partial D / \partial x$ and $\partial D / \partial y$. Repetition of this process will give the final solution of the problem.

On the balance of acting forces at the equator. It has been customary in most cases to neglect the terms of field accelerations or the non-linear terms. This seems to be possible except for narrow equatorial zones. However, the Coriolis forces vanish or become vanishingly small exactly at or very close to the equator, so that these non-linear terms are considered to balance the pressure gradients and are no longer negligible. We may suppose that $-w(\partial u / \partial z)$ and $-w(\partial v / \partial z)$ are small compared with $-v(\partial u / \partial y)$ and $-v(\partial v / \partial y)$ which have to balance the pressure gradients without the Coriolis forces at the equator. In a geostrophic computation, it is assumed that several terms in the dynamical equations except the Coriolis forces and the pressure gradients are negligibly small. Thus we have $2 \omega \sin \varphi v$ $=\partial D / \partial x ;-2 \omega \sin \varphi u=\partial D / \partial y$. This balance evidently fails at the equator where the Coriolis forces vanish. For this reason we have to consider a factor $f$ which would balance the pressure gradients at and near the equator, but becomes much smaller than the Coriolis terms at higher latitudes. Thus, in a meridional direction, we must have

$$
-2 \omega \sin \varphi u+f=\partial D / \partial y \text {. }
$$

$f$ will not vanish even if the Coriolis term vanishes at the equator, thus balancing the pressure gradient $\partial D / \partial y$ there.

There is another requirement imposed on $f$. According to Hidaka ${ }^{2}$ and Tsuchiya, ${ }^{5)}$ the equatorial velocity $u$ can be computed by differentiating the geostrophic equations with respect to $y$ and putting $\varphi=y=0$. The approximate validity of the resulting formula

$$
u_{0}=(R / 2 \omega)\left(\partial^{2} D / \partial y^{2}\right)_{\varphi=0},
$$

where $R$ is the average radius of the Earth, is now widely accepted. In order that this requirement be fulfilled, however, $\partial f / \partial y$ should become much smaller than $(\partial / \partial y)(-2 \omega \sin \varphi u)$ at the equator. This is another necessary condition which $f$ has to satisfy. 
Accordingly, $f$ in (4) should be in a balance with $\partial D / \partial y$ at the equator, and its derivative $\partial f / \partial y$ must either be small or vanish there, and the zonal velocity component $u$ may be distributed symmetrically around the equator. This can be seen from the meridional section of the equatorial undercurrent recently published by Knauss (1959). On the other hand, we have a very little knowledge on the meridional distribution of the meridional component $v$. We have not had an exact image on this question as yet. However, it can be anticipated that $v$ as well as $\partial v / \partial y$ will not vanish even at the equator itself. Thus it seems reasonable for us to suppose that $-v(\partial v / \partial y)$ corresponds with or with a part of $f$ which we have considered above. It will be a small quantity at or very near the equator, being in balance with $\partial D / \partial y$ which in turn appears quite small there. Thus in a region very close to the equator, $-2 \omega \sin \varphi u$ is small, but its meridional gradient: $\left|(\partial / \partial y)(-2 \omega \sin \varphi u)_{\varphi=0}\right|$ $=(2 \omega / R)\left|u_{0}\right|$ can be much larger than $\left|(\partial / \partial y)(-v(\partial v / \partial y))_{\varphi=0}\right|=\mid\left(1 / 2 \cdot \partial^{2} v^{2}\right.$ $\left.\mid \partial y_{2}\right)_{\varphi=0} \mid$ which is the meridional gradient of an inertia term.

It is quite hard to know the distribution of meridional gradient $\partial D / \partial y$ at the equator. But various estimations show that $\partial D / \partial y$ never vanishes there, though it is a small quantity (Ca. $10^{-5}$ c.g.s.).

So we may suppose that, in the equation $-2 \omega \sin \varphi u-v(\partial v / \partial y)=$ $\partial D / \partial y,-2 \omega \sin \varphi u$ vanishes at the equator, thus a balance having to exist primarily between $-v(\partial v / \partial y)$ and $\partial D / \partial y$ there, whereas the derivative of the Coriolis force $-2 \omega \sin \varphi u$ is much larger than that of the inertia term $-v(\partial v / \partial y)$, thus an approximate balance between $\partial / \partial y(-2 \omega \sin \varphi u)$ and $(\partial / \partial y)(\partial D / \partial y)$ at the equator.

In this paper, the terms of both horizontal and vertical mixing were neglected, though some of them appear to act as a part of $f$ in (4). The importance of these terms will be left for later studies.

Solution of the non-linear equations for equatorial flows. Neglecting $-w(\partial u / \partial z)$ and $-w(\partial v / \partial z)$ as small, we have (3) from (1). Consider that $\partial D / \partial x, \partial D / \partial y$ and $u, v$ can be expanded in series of $y$ around the equator $\varphi=0$ or $y=0$. Thus, it may be assumed that

$$
\begin{aligned}
& \frac{\partial D}{\partial y}=d_{0}+d_{1} y+d_{2} y^{2}+\cdots, \\
& \frac{\partial D}{\partial y}=\delta_{0}+\delta_{1} y+\delta_{2} y^{2}+\cdots,
\end{aligned}
$$

where, $d_{0}, d_{1}, d_{2}, \ldots ; \delta_{0}, \delta_{1}, \delta_{2}, \cdots$, can be obtained from the meridional distribution of the geopotential distance $D$. Further let $u$ and $v$ be given by

$$
u=u_{0}+u^{\prime} y+\frac{u^{\prime \prime}}{2 !} y^{2}+\cdots+\frac{u^{(n)}}{n !} y^{n}+\cdots
$$




$$
u=v_{0}+v^{\prime} y+\frac{v^{\prime \prime}}{2 !} y^{2}+\cdots+\frac{v^{(n)}}{n !} y^{n}+\cdots,
$$

where $u_{0}, v_{0}$ are the east and north component of the current velocity at the equator, whereas $u^{\prime}, u^{\prime \prime}, \cdots, u^{(n)}, \cdots ; v^{\prime}, v^{\prime \prime}, \cdots, v^{(n)}, \cdots$ are the 1st, 2 nd, $\cdots, n$th derivatives of $u$ and $v$ at $y=0$, which are to be determined by solving (3).

Substituting (6)-(9) in (3), we have

$$
\begin{aligned}
\left(\frac{2 \omega}{R} y\right. & \left.-2 \omega \frac{y^{3}}{3 ! R^{3}}+\cdots\right)\left(v_{0}+v^{\prime} y+\frac{v^{\prime \prime}}{2 !} y^{2}+\cdots+\frac{v^{(n)}}{n !} y^{n}+\cdots\right) \\
& -\left(v_{0}+v^{\prime} y+\frac{v^{\prime \prime}}{2 !} y^{2}+\cdots+\frac{v^{(n)}}{n !} y^{n}+\cdots\right) \\
& \times\left\{u^{\prime}+u^{\prime \prime} y+\frac{u^{\prime \prime \prime}}{2 !} y^{2}+\cdots+\frac{u^{(n-1)}}{(n-2) !} y^{n-2}+\frac{u^{(n)}}{(n-1) !} y^{n-1}+\cdots\right\} \\
= & d_{0}+d_{1} y+d_{2} y^{2}+\cdots
\end{aligned}
$$

or

$2 \omega \sin \varphi v-v \frac{\partial u}{\partial y}-\frac{\partial D}{\partial x}=\left(-u^{\prime} v_{0}-d_{0}\right)+\left(\frac{2 \omega}{R} v_{0}-u^{\prime} v^{\prime}-u^{\prime \prime} v_{0}-d_{1}\right) y$

$$
\begin{aligned}
& +\left(\frac{2 \omega}{R} v^{\prime}-\frac{1}{2 !} u^{\prime} v^{\prime \prime}-u^{\prime \prime} v^{\prime}-\frac{1}{2 !} u^{\prime \prime \prime} v_{0}-d_{2}\right) y^{2} \\
& +\left(\frac{2 \omega}{R} v^{(n-1)}-\frac{2 \omega}{R} \cdot \frac{1}{3 ! R^{2}} v^{(n-3)}-\frac{1}{n !} u^{\prime} v^{(n)}-\cdots\right. \\
& \left.-\frac{1}{(n-1) !} u^{(n)} v^{\prime}-\frac{1}{n !} u^{(n+1)} v_{0}-d_{n}\right) y^{n}+\cdots=0
\end{aligned}
$$

Equating to zero the coefficients of $y^{0}, y, y^{2}, y^{3}, \cdots, y^{n} \cdots$, we have

$$
\begin{aligned}
& -u^{\prime} v_{0}-d_{0}=0, \\
& \frac{2 \omega}{R} v_{0}-u^{\prime} v^{\prime}-u^{\prime \prime} v_{0}-d_{1}=0, \\
& \quad \ldots \ldots \ldots \cdots \cdots \\
& \frac{1}{(n-1) !} \cdot \frac{2 \omega}{R} v^{(n-1)}-\frac{1}{(n-2) !} \frac{2 \omega}{R} v^{(n-3)}-\cdots \\
& \quad-\frac{1}{n !} u^{\prime} v^{(n)}-\cdots-\frac{1}{(n-1) !} u^{(n)} v^{\prime}-\frac{1}{n !} u^{(n+1)} v_{0}-d_{n}=0 .
\end{aligned}
$$

In a similar manner, we have from the second equation of (3),

$$
\begin{aligned}
& -v_{0} v^{\prime}-\delta_{0}=0 \\
& \frac{2 \omega}{R} u_{0}-v_{0} v^{\prime \prime}-v^{\prime}-\delta_{1}=0 \\
& \quad \ldots \ldots \ldots \ldots \ldots \\
& -\frac{1}{(n-1) !} \cdot \frac{2 \omega}{R} \cdot u^{(n-1)}+\frac{1}{(n-3) !} \frac{2 \omega}{R} \cdot \frac{1}{6 R^{2}} u^{(n-3)}-\cdots \\
& \quad-\frac{1}{n !} v_{0} v^{(n-1)}-\cdots-\frac{1}{(n-1) !} v_{0} v^{(n)}-\frac{1}{n !} v_{0} v^{(n+1)}-\delta_{n}=0
\end{aligned}
$$

Two sets of (12) and (13) are non-linear and simultaneous. 
They satisfy the expansions of (13) to $y^{n}$. Here we notice, however, that the number of the equations in (12) and (13) is $2 n+2$, while we have $2 n+4$ unknowns, we have therefore a larger number of unknowns than that of the equations. For this reason, these equations cannot be solved in their forms as they stand.

A feasible countermeasure for this situation will be to obtain approximate roots by neglecting $-(1 / n !) u^{(n+1)} v_{0}$ and $-(1 / n !) v_{0} v^{(n+1)}$ in the last equations of (12) and (13). By neglecting these terms, the number of the unknowns just equals that of the equations. It is therefore possible to solve the equations provided the expansions are satisfied only up to $y^{n}$, having the residues

$$
-(1 / n !) u^{(n+1)} v_{0} y^{n+1} \text { and }-(1 / n !) v_{0} v^{(n+1)} y^{n+1}
$$

respectively. Actually there is a good reason for us to neglect (14), because (14) become smaller and smaller as $n$ increases. The result will be therefore more improved as we adopt a larger number of equations. Thus we have to try several approximations, of which we shall mention the cases $n=2$ and 3. 1) Case $n=2$. We have

$-u^{\prime} v_{0}-d_{0}=0, \quad-v_{0} v^{\prime}-\delta_{0}=0$,

$(2 \omega / R) v_{0}-u^{\prime} v^{\prime}-u^{\prime \prime} v_{0}-d_{1}=0, \quad-(2 \omega / R) u_{0}-\left(v^{\prime}\right)^{2}-v_{0} v^{\prime \prime}-\delta_{1}=0$,

$(2 \omega / R) v^{\prime}-(1 / 2) u^{\prime} v^{\prime \prime}-u^{\prime \prime} v^{\prime}-d_{2}=0, \quad-(2 \omega / R) u^{\prime}-(3 / 2) v^{\prime} v^{\prime \prime}-\delta_{2}=0$.

Eliminating $v^{\prime}, v^{\prime \prime}$ and $u^{\prime}, u^{\prime \prime}$ out of the five equations without $u_{0}$, we have a quartic equations for $v_{0}$, or

$$
\left(3 d_{2} \delta_{0}-d_{0} \delta_{2}\right) v_{0}^{4}+(2 \omega / R) d_{0}^{2} v_{0}^{3}+3 d_{1} \delta_{0}^{2} v_{0}^{2}+3 d_{0} \delta_{0}^{3}=0 .
$$

(16) will give the possible value of $v_{0}$ as one of its four roots. Substituting $v_{0}$ thus obtained in the remaining equation of (15) with $u_{0}$, we have

$$
u_{0}=-(2 \omega / R)\left(\left(2 d_{0} / d_{0}\right) v_{0}^{3}+\left(2 d_{1} \delta_{0} / d_{0}\right) v_{0}+\delta_{1}+3\left(\delta_{0}^{2} / v_{0}^{2}\right)\right)
$$

In this case, the expansions of (3) will be satisfied up to $y$ and the residues will be $-1 / 2 u^{\prime \prime} v_{0} y^{2}$ and $-1 / 2 v_{0} v^{\prime \prime} y^{2}$ respectively.

2) Case $n=3$. The equations to be solved are

$$
\begin{aligned}
& -u^{\prime} v_{0}-d_{0}=0, \quad \frac{2 \omega}{R} v_{0}-u^{\prime} v^{\prime}-u^{\prime \prime} v_{0}-d_{1}=0, \\
& \frac{2 \omega}{R} v^{\prime}-\frac{1}{2} u^{\prime} v^{\prime \prime}-u^{\prime \prime} v^{\prime}-\frac{1}{2} u^{\prime \prime \prime} v_{0}-d_{2}=0, \\
& \frac{1}{2} \cdot \frac{2 \omega}{R} v^{\prime \prime}-\frac{2 \omega}{R} \cdot \frac{1}{6 R^{2}} v_{0}-\frac{1}{6} u^{\prime} v^{\prime \prime \prime}-u^{\prime \prime} v^{\prime \prime}-\frac{1}{2} u^{\prime \prime \prime} v^{\prime}-d_{3}=0 \\
& -v_{0} v^{\prime}-\delta_{0}=0, \quad-\frac{2 \omega}{R} u_{0}-v_{0} v^{\prime \prime}-\left(v^{\prime}\right)^{2}-\delta_{1}=0, \\
& -\frac{2 \omega}{R} u^{\prime}-\frac{3}{2} v^{\prime} v^{\prime \prime}-\frac{1}{2} v_{0} v^{\prime \prime \prime}-\delta_{2}=0, \\
& -\frac{1}{2} \cdot \frac{2 \omega}{R} u^{\prime \prime}-\frac{2 \omega}{R} \cdot \frac{1}{6 R^{2}} u_{0}-\frac{2}{3} v^{\prime} v^{\prime \prime \prime}-\frac{1}{2}\left(v^{\prime \prime}\right)^{2}=0 .
\end{aligned}
$$

In this case, the expansions of (3) will be satisfied up to $y^{2}$ and the 
residues will be $-1 / 2 u^{\prime \prime} v_{0} y^{3}$ and $u_{0} v^{\prime \prime} y^{3}$ respectively. The resulting equation giving $v_{0}$ is

$$
\begin{aligned}
- & \frac{1}{288 R^{4}} \cdot\left(\frac{2 \omega}{R}\right)^{2} v_{0}^{14}-\frac{2 \omega}{R}\left(\frac{d_{1}}{288 R^{4}}+\frac{d_{3}}{24 R^{2}}\right) v_{0}^{13}-\left(\frac{d_{1} d_{3}}{48 R^{2}}+\frac{d_{3}^{2}}{8}\right) v_{0}^{12} \\
& -\frac{2 \omega}{R}\left(\frac{d_{0} \delta_{0}}{96 R^{4}}+\frac{d_{0} \delta_{2}}{72 R^{2}}+\frac{d_{2} \delta_{0}}{24 R^{2}}\right) v_{0}^{11}+\left\{\left(\frac{2 \omega}{R}\right)^{2} \cdot\left(\frac{d_{0}^{2}}{72 R^{2}}-\frac{d_{1}^{2}}{32}\right)\right. \\
& \left.-\left(\frac{d_{0} d_{3} \delta_{0}}{16 R^{2}}+\frac{d_{0} d_{1} \delta_{2}}{144 R^{2}}+\frac{d_{1} d_{2} \delta_{0}}{48 R^{2}}+\frac{d_{1}^{2} \delta_{1}}{96 R^{2}}+\frac{d_{1}^{2} \delta_{3}}{16}+\frac{d_{2} d_{3} \delta_{0}}{4}+\frac{d_{0} d_{3} \delta_{2}}{12}\right)\right\} v_{0}^{10} \\
& +\frac{2 \omega}{R}\left(\frac{d_{0}^{2} d_{1}}{144 R^{2}}+\frac{d_{1}^{3}}{32}+\frac{d_{0}^{2} d_{3}}{12}\right) v_{0}^{9}+\left\{-\frac{3 d_{0} d_{1} \delta_{0}}{16} \cdot\left(\frac{2 \omega}{R}\right)^{2}-\frac{d_{0}^{2} \delta_{0} \delta_{2}}{48 R^{2}}\right. \\
& -\frac{d_{0} d_{2} \delta_{0}^{2}}{16 R^{2}}-\frac{d_{1}^{2} \delta_{0}^{2}}{32 R^{2}}-\frac{d_{0} d_{1} \delta_{0} \delta_{1}}{16 R^{2}}-\frac{d_{1}^{2} \delta_{0} \delta_{2}}{12}-\frac{3 d_{0} d_{1} \delta_{0} \delta_{3}}{8}-\frac{d_{0}^{2} \delta_{2}^{2}}{72}-\frac{d_{2}^{2} \delta_{0}^{2}}{8} \\
& \left.-\frac{d_{0} d_{2} \delta_{0} \delta_{2}}{12}\right\} v_{0}^{8}+\frac{2 \omega}{R}\left(\frac{d_{0}^{3} \delta_{0}}{48 R^{2}}+\frac{d_{0} \delta_{0}^{3}}{12 R^{2}}+\frac{29 d_{0} d_{1}^{2} \delta_{0}}{96}+\frac{d_{0}^{2} d_{2} \delta_{0}}{12}+\frac{d_{0}^{3} \delta_{2}}{36}\right) v_{0}^{7} \\
& +\left\{\left(\frac{2 \omega}{R}\right)^{2} \cdot\left(-\frac{9 d_{0}^{2} \delta_{0}^{2}}{32}-\frac{d_{0}^{4}}{72}\right)-\frac{9 d_{0}^{2} \delta_{0}^{2} d_{3}}{16}-\frac{3 d_{0}^{2} \delta_{0}^{2} \delta_{1}}{32 R^{2}}-\frac{d_{0} d_{1} \delta_{0}^{3}}{12 R^{2}}\right. \\
& \left.-\frac{d_{0} d_{1} \delta_{0}^{2} \delta_{2}}{12}+\frac{d_{0} d_{3} \delta_{0}^{3}}{2}\right\} v_{0}^{6}+\frac{2 \omega}{R} \cdot \frac{31}{32} d_{0}^{2} d_{1} \delta_{0}^{2} v_{0}^{5}+\left(-\frac{5 d_{0}^{2} \delta_{0}^{4}}{32 R^{2}}+\frac{d_{1}^{2} \delta_{0}^{4}}{4}\right. \\
& \left.-\frac{7 d_{0}^{2} \delta_{0}^{3} \delta_{2}}{12}+\frac{d_{0} d_{2} \delta_{0}^{4}}{2}\right) v_{0}^{4}+\frac{2 \omega}{R} \cdot \frac{83}{96} d_{0}^{3} \delta_{0}^{3} v_{0}^{3}+\frac{3 d_{0} d_{1} \delta_{0}^{5}}{4} v_{0}^{2}+\frac{5 d_{0}^{2} \delta_{0}^{6}}{8}=0
\end{aligned}
$$

If we evaluate $v_{0}$ by solving (19), $u_{0}$ will be given by

$$
\begin{aligned}
u_{0}= & -\frac{2 \omega}{R} \cdot \frac{1}{3 d_{0} \delta_{0} v_{0}^{2}+d_{1} v_{0}^{4}}\left\{\frac{1}{3 R^{2}} \cdot \frac{2 \omega}{R} \cdot v_{0}^{7}-2 d_{3} \cdot v_{0}^{6}\right. \\
& +\left(\frac{2}{3} d_{0} \delta_{2}+d_{1} \delta_{1}+2 d_{2} \delta_{0}\right) v_{0}^{4}-\frac{2}{3} \cdot \frac{2 \omega}{R} \cdot d_{0}^{2} \cdot v_{0}^{3} \\
& \left.+\left(3 d_{1} \delta_{0}^{2}+3 d_{0} \delta_{0} \delta_{1}\right) v_{0}^{2}+5 d_{0} \delta_{0}^{3}\right\} .
\end{aligned}
$$

Further approximations are of course possible theoretically. But the derivation of the equation giving $v_{0}$ will be extremely complicated then, so that we have to satisfy ourselves only with this degree of approximation. The accuracy of the solution will be estimated by a comparison with the results of the cases $n=2$ and $n=3$.

( To be continued)

\section{References}

1) Arthur, S. (1960): Deep-Sea Research, 6, 287-297.

2) Hidaka, K. (1955) : Japan. J. Geophysics, 1 (2), 56-60.

3) (1961): Records of Oceanogr. Works in Japan.

4) Hidaka, K., and Y. Nagata (1958): Geophys. J. Roy. Astron. Soc., 1 (3), 198-207.

5) Tsuchiya, M. (1955) : Records of Oceanogr. Works in Japan, 2(2), 1-6. 